\title{
Basic science (November 2006)
}

1. Acconcia F, Manavathi B, Mascarenhas J, Talukder MH, Mills G, Kumar R. An inherent role of integrin-linked kinase-estrogen receptor alpha interaction in cell migration. Cancer Res 2006; 66: 11030-11038.

2. Adly L, Hill D, Sherman ME, Sturgeon SR, Fears T, Mies C, Ziegler RG, Hoover RN, Schairer C. Serum concentrations of estrogens, sex hormonebinding globulin, and androgens and risk of breast cancer in postmenopausal women. Int $J$ Cancer 2006; 119: 2402-2407.

3. Arslan AA, Zeleniuch-Jacquotte A, Lukanova A, Afanasyeva Y, Katz J, Levitz M, Del Priore G, Toniolo P. Effects of parity on pregnancy hormonal profiles across ethnic groups with a diverse incidence of breast cancer. Cancer Epidemiol BiomarkPrevent 2006; 15: 2123-2130.

4. Bernstein JL, Teraoka S, Southey MC, Jenkins MA, Andrulis IL, Knight JA, John EM, Lapinski R, Wolitzer AL, Whittemore AS, West D, Seminara $D$, Olson ER, Spurdle AB, ChenevixTrench G, Giles GG, Hopper JL, Concannon P. Population-based estimates of breast cancer risks associated with ATM gene variants c.7271T $>$ G and c.1066-6T $>$ G (IVS10-6T $>$ G) from the breast cancer family registry. Hum Mutat 2006; 27: 1122-1128.

5. Bistulfi G, Pozzi S, Ren MQ, Rossetti S, Sacchi N. A repressive epigenetic domino effect confers susceptibility to breast epithelial cell transformation: implications for predicting breast cancer risk. Cancer Res 2006; 66: 10308-10314.

6. Boyd NF, Martin LJ, Sun LM, Guo H, Chiarelli A, Hislop G, Yaffe M, Minkini S. Body size, mammographic density, and breast cancer risk. Cancer Epidemiol BiomarkPrevent 2006; 15: 2086-2092.

7. Brantley-Sieders DM, Fang WB, Hwang Y, Hicks $D$, Chen J. Ephrin-A1 facilitates mammary tumor metastasis through an angiogenesis-dependent mechanism mediated by EphA receptor and vascular endothelial growth factor in mice. Cancer Res 2006; 66: 10315-10324.

8. Brennan DJ, Jirstrom K, Kronblad A, Millikan RC, Landberg G, Duffy MJ, Ryden L, Gallagher WM,

BCO/635/2007/JW
O'Brien SL. CA IX is an independent prognostic marker in premenopausal breast cancer patients with one to three positive lymph nodes and a putative marker of radiation resistance. Clin Cancer Res 2006; 12: 6421-6431.

9. Budd GT, Cristofanilli M, Ellis MJ, Stopeck A, Borden E, Miller MC, Matera J, Repollet M, Doyle GV, Terstappen L, Hayes DF. Circulating tumor cells versus imaging - Predicting overall survival in metastatic breast cancer. Clin Cancer Res 2006; 12: 6403-6409.

10. Byun HJ, Hong IK, Kim E, Jin YJ, Jeoung DI, Hahn JH, Kim YM, Park SH, Lee H. A splice variant of CD99 increases motility and MMP-9 expression of human breast cancer cells through the AKT-, ERK-, and JNK-dependent AP-1 activation signaling pathways. J Biol Chem 2006; 281: 34833-34847.

11. den Hollander P, Rayala SK, Coverley D, Kumar R. Ciz1, a novel DNA-binding coactivator of the estrogen receptor alpha, confers hypersensitivity to estrogen action. Cancer Res 2006; 66: 11021-11029.

12. Derksen PWB, Liu XL, Saridin F, van der Gulden $H$, Zevenhoven J, Evers B, van Beijnum JR, Griffioen AW, Vink J, Krimpenfort P, Peterse JL, Cardiff RD, Berns A, Jonkers J. Somatic inactivation of E-cadherin and p53 in mice leads to metastatic lobular mammary carcinoma through induction of anoikis resistance and angiogenesis. Cancer Cell 2006; 10: 437-449.

13. Dolled-Filhart M, Ryden L, Cregger M, Jirstrom K, Harigopal M, Camp RL, Rimm DL. Classification of breast cancer using genetic algorithms and tissue microarrays. Clin Cancer Res 2006; 12: 6459-6468.

14. Du ZJ, Podsypanina K, Huang SX, McGrath A, Toneff MJ, Bogoslovskaia E, Zhang XM, Moraes RC, Fluck M, Allred DC, Lewis MT, Varmus HE, Li Y. Introduction of oncogenes into mammary glands in vivo with an avian retroviral vector initiates and promotes carcinogenesis in mouse models. Proc Natl Acad Sci USA 2006; 103: 17396-17401.

15. Filardo EJ, Graeber CT, Quinn JA, Resnick MB, Giri D, DeLellis RA, Steinhoff MM, Sabo E. 
Distribution of GPR30, a seven membranespanning estrogen receptor, in primary breast cancer and its association with clinicopathologic determinants of tumor progression. Clin Cancer Res 2006; 12: 6359-6366.

16. Gallet M, Mentaverri R, Sevenet N, Brazier M, Kamel S. Ability of breast cancer cell lines to stimulate bone resorbing activity of mature osteoclasts correlates with an anti-apoptotic effect mediated by macrophage colony stimulating factor. Apoptosis 2006; 11: 1909-1921.

17. Gonzalez-Santiago L, Suarez Y, Zarich N, Munoz-Alonso MJ, Cuadrado A, Martinez T, Goya L, Iradi A, Saez-Tormo G, Maier JV, Moorthy A, Cato ACB, Rojas JM, Munoz A. Aplidin (R) induces JNK-dependent apoptosis in human breast cancer cells via alteration of glutathione homeostasis, Rac1 GTPase activation, and MKP-1 phosphatase downregulation. Cell Death Differ 2006; 13: 1968-1981.

18. Hicks DG, Yoder BJ, Short S, Tarr S, Prescott N, Crowe JP, Dawson AE, Budd GT, Sizemore S, Cicek M, Choueiri TK, Tubbs RR, Gaile D, Nowak N, Accavitti-Loper M, Frost AR, Welch DR, Casey G. Loss of breast cancer metastasis suppressor 1 protein expression predicts reduced disease-free survival in subsets of breast cancer patients. Clin Cancer Res 2006; 12: 6702-6708.

19. Hossain A, Kuo MT, Saunders GF. Mir-17-5p regulates breast cancer cell proliferation by inhibiting translation of AlB1 mRNA. Mol Cell Biol 2006; 26: 8191-8201.

20. Hu JC, Booth MJ, Tripuraneni G, Zaidi SAA, de Bella MT, Slade MJ, Marley SB, Gordon MYA, Coffin RS, Coombes RC, Kamalati T. A novel HSV-1 virus, JS1/34.5(-)/47(-), purges contaminating breast cancer cells from bone marrow. Clin Cancer Res 2006; 12: 6853-6862.

21. Hui L, Zheng Y, Yan Y, Bargonetti J, Foster DA. Mutant p53 in MDA-MB-231 breast cancer cells is stabilized by elevated phospholipase $D$ activity and contributes to survival signals generated by phospholipase D. Oncogene 2006; 25: 7305-7310.

22. Husen B, Huhtinen K, Saloniemi T, Messinger J, Thole HH, Poutanen M. Human hydroxysteroid (17-beta) dehydrogenase 1 expression enhances estrogen sensitivity of MCF-7 breast cancer cell xenografts. Endocrinology 2006; 147: 5333-5339.

23. Ivshina AV, George J, Senko O, Mow B, Putti TC, Smeds J, Lindahl T, Pawitan Y, Hall P, Nordgren $\mathrm{H}$, Wong JEL, Liu ET, Bergh J, Kuznetsov VA, Miller LD. Genetic reclassification of histologic grade delineates new clinical subtypes of breast cancer. Cancer Res 2006; 66: 10292-10301.
24. Jang JW, Boxer RB, Chodosh LA. Isoform-specific ras activation and oncogene dependence during MYC- and Wnt-induced mammary tumorigenesis. Mol Cell Biol 2006; 26: 8109-8121.

25. Jasienska G, Kapiszewska M, Ellison PT, Kalemba-Drozdz M, Nenko I, Thune I, Ziomkiewicz A. CYP17 genotypes differ in salivary 17-beta estradiol levels: a study based on hormonal profiles from entire menstrual cycles. Cancer Epidemiol Biomark Prevent 2006; 15: 2131-2135.

26. Karppinen SM, Barkardottir RB, Backenhorn K, Sydenham T, Syrjakoski K, Schleutker J, Ikonen T, Pylkas K, Rapakko K, Erkko H, Johannesdottir G, Gerdes AM, Thomassen M, Agnarsson BA, Grip M, Kallioniemi A, Kere J, Aaltonen LA, Arason A, Moller P, Kruse TA, Borg A, Winqvist R. Nordic collaborative study of the BARD1 Cys557Ser allele in 3956 patients with cancer: enrichment in familial BRCA1/BRCA2 mutationnegative breast cancer but not in other malignancies. J Med Genet 2006; 43: 856-862.

27. Kim DS, Park SS, Nam BH, Kim IH, Kim SY. Reversal of drug resistance in breast cancer cells by transglutaminase 2 inhibition and nuclear factor-kappa B inactivation. Cancer Res 2006; 66: 10936-10943.

28. Kim EHJ, Willett WC, Colditz GA, Hankinson SE, Stampfer MJ, Hunter DJ, Rosner B, Holmes MD. Dietary fat and risk of postmenopausal breast cancer in a 20-year follow-up. Am J Epidemiol 2006; 164: 990-997.

29. Lewis SJ, Harbord RM, Harris R, Smith GD. Meta-analyses of observational and genetic association studies of folate intakes or levels and breast cancer risk. J Natl Cancer Inst 2006; 98: 1607-1622.

30. Lindvall C, Evans NC, Zylstra CR, Li Y, Alexander CM, Williams BO. The Wnt signaling receptor Lrp5 is required for mammary ductal stem cell activity and Wnt1-induced tumorigenesis. J Biol Chem 2006; 281: 35081-35087.

31. Loladze AV, Stull MA, Rowzee AM, DeMarco J, Lantry JH, Rosen CJ, LeRoith D, Wagner KU, Hennighausen L, Wood TL. Epithelial-specific and stage-specific functions of insulin-like growth factor-I during postnatal mammary development. Endocrinology 2006; 147: 5412-5423.

32. Long JR, Kataoka N, Shu XO, Wen WQ, Gao YT, Cai QY, Zheng W. Genetic polymorphisms of the CYP19A1 gene and breast cancer survival. Cancer Epidemiol BiomarkPrevent 2006; 15: 2115-2122.

33. Milanese TR, Hartmann LC, Sellers TA, Frost MH, Vierkant RA, Maloney SD, Pankratz VS, Degnim AC, Vachon CM, Reynolds CA, Thompson RA, 
Melton LJ, Goode EL, Visscher DW. Age-related lobular involution and risk of breast cancer. J Natl Cancer Inst 2006; 98: 1600-1607.

34. Mussi P, Liao L, Park SE, Ciana P, Maggi A, Katzenellenbogen BS, Xu JM, O'Malley BW. Haploinsufficiency of the corepressor of estrogen receptor activity (REA) enhances estrogen receptor function in the mammary gland. Proc Natl Acad Sci USA 2006; 103: 16716-16721.

35. Novak P, Jensen T, Oshiro MM, Wozniak RJ, Nouzova M, Watts GS, Klimecki WT, Kim C, Futscher BW. Epigenetic inactivation of the HOXA gene cluster in breast cancer. Cancer Res 2006; 66: 10664-10670.

36. Potti A, Dressman HK, Bild A, Riedel RF, Chan G, Sayer R, Cragun J, Cottrill $H$, Kelley MJ, Petersen R, Harpole D, Marks J, Berchuck A, Ginsburg GS, Febbo P, Lancaster J, Nevins JR. Genomic signatures to guide the use of chemotherapeutics. Nat Med 2006; 12: 1294-1300.

37. Qian CN, Berghuis B, Tsarfaty G, Bruch M, Kort EJ, Ditlev J, Tsarfaty I, Hudson E, Jackson DG, Petillo D, Chen JD, Resau JH, Teh BT. Preparing the "soil": the primary tumor induces vasculature reorganization in the sentinel lymph node before the arrival of metastatic cancer cells. Cancer Res 2006; 66: 10365-10376.

38. Reeves GK, Beral V, Green J, Gathani T, Bull D. Hormonal therapy for menopause and breastcancer risk by histological type: a cohort study and meta-analysis. Lancet Oncol 2006; 7: 910-918.

39. Regan MM, Viale G, Mastropasqua MG, Maiorano E, Golouh R, Carbone A, Brown B, Suurkula M, Langnan G, Mazzucchelli L, Braye S, Grigolato P, Gelber RD, Castiglione-Gertsch M, Price KN, Coates AS, Goldhirsch A, Gusterson B. Re-evaluating adjuvant breast cancer trials: assessing hormone receptor status by immunohistochemical versus extraction assays. $J$ Natl Cancer Inst 2006; 98: 1571-1581.

40. Shyamala G, Chou YC, Cardiff RD, Vargis E. Effect of c-neu/ErbB2 expression levels on estrogen receptor alpha-dependent proliferation in mammary epithelial cells: implications for breast cancer biology. Cancer Res 2006; 66: 10391-10398.

41. Smith APL, Henze M, Lee JA, Osborn KG, Keck JM, Tedesco D, Bortner DM, Rosenberg MP, Reed SI. Deregulated cyclin E promotes p53 loss of heterozygosity and tumorigenesis in the mouse mammary gland. Oncogene 2006; 25: 7245-7259.

42. Song H, Ki SH, Kim SG, Moon A. Activating transcription factor 2 mediates matrix metalloproteinase-2 transcriptional activation induced by p38 in breast epithelial cells. Cancer Res 2006; 66: 10487-10496.

43. Sorlie T, Perou CM, Fan C, Geisler S, Aas T, Nobel A, Anker G, Akslen LA, Botstein D, Borresen-Dale AL, Lonning PE. Gene expression profiles do not consistently predict the clinical treatment response in locally advanced breast cancer. Mol Cancer Ther 2006; 5: 2914-2918.

44. Sotgia F, Rui H, Bonuccelli G, Mercier I, Pestell RG, Lisanti MP. Caveolin-1, mammary stem cells, and estrogen-dependent breast cancers. Cancer Res 2006; 66: 10647-10651.

45. Sternlicht MD, Dunning AM, Moore DH, Pharoah PDP, Ginzinger DG, Chin K, Gray JW, Waldman FM, Ponder BAJ, Werb Z. Prognostic value of PAl1 in invasive breast cancer: evidence that tumor-specific factors are more important than genetic variation in regulating PAl1 expression. Cancer Epidemiol BiomarkPrevent 2006; 15: 2107-2114.

46. Wang X, Zhou YX, Qiao W, Tominaga Y, Ouchi M, Ouchi T, Deng CX. Overexpression of aurora kinase $A$ in mouse mammary epithelium induces genetic instability preceding mammary tumor formation. Oncogene 2006; 25: 7148-7158.

47. Yehiely F, Moyano JV, Evans JR, Nielsen TO, Cryns VL. Deconstructing the molecular portrait of basal-like breast cancer. Trends $\mathrm{Mol} \mathrm{Med}$ 2006; 12: 537-544.

Prepared by R. Sutherland, J. Scorer Cancer Research Program Garvan Institute of Medical Research Darlinghurst, NSW, Australia 